\title{
Impacto económico de la resistencia a la meticilina en pacientes con bacteriemia por Staphylococcus aureus en hospitales de Bogotá
}

\author{
Liliana I. Barrero ${ }^{1,2}$, Juan S. Castillo1,2, Aura L. Leal ${ }^{1,2,3}$, Ricardo Sánchez ${ }^{1,2}$, Jorge A. Cortés ${ }^{1,2,4}$, \\ Carlos A. Álvarez ${ }^{1,2}$, Andrés L. González ${ }^{1,2}, \mathrm{GREBO}^{2}$ \\ 1 Grupo de Investigación en Enfermedades Infecciosas, Facultad de Medicina, Universidad Nacional de \\ Colombia, Bogotá, D.C., Colombia \\ 2 Grupo para el Control de la Resistencia Bacteriana de Bogotá (GREBO), Bogotá, D.C., Colombia \\ ${ }^{3}$ Departamento de Microbiología, Facultad de Medicina, Universidad Nacional de Colombia, Bogotá, D.C., \\ Colombia \\ ${ }^{4}$ Departamento de Medicina Interna, Facultad de Medicina, Universidad Nacional de Colombia, Bogotá, D.C., \\ Colombia
}

Introducción. Las infecciones por microorganismos resistentes, especialmente las que involucran el torrente sanguíneo, se asocian a un mayor uso de recursos. Sus estimaciones son variables y dependen de la metodología utilizada. Staphylococcus aureus es el agente de sangre aislado con mayor frecuencia en nuestro medio. No existe información sobre el costo asociado con la atención de bacteriemias por $S$. aureus resistente a meticilina en nuestro país.

Objetivo. Presentar una aproximación del costo de atención de las bacteriemias por $S$. aureus resistente a la meticilina en nueve hospitales de Bogotá.

Materiales y métodos. Se incluyeron 204 pacientes en un estudio de cohortes multicéntrico en una razón de 1:1 según la resistencia. Se aproximaron los costos médicos directos con base en las facturas del período de hospitalización; en cuanto al período de la bacteriemia, los costos detallados se calcularon aplicando las tarifas estandarizadas.

Resultados. No se encontraron diferencias significativas en las características clínicas y demográficas de los grupos, salvo en los antecedentes de la bacteriemia. El 53 \% de los sujetos falleció durante la hospitalización. La estancia y el valor total facturado por la hospitalización fueron significativamente mayores en el grupo con bacteriemia por $S$. aureus resistente a la meticilina, así como los costos de la estancia en cuidados intensivos, de los antibióticos, los líquidos parenterales, los exámenes de laboratorio y la terapia respiratoria. El incremento crudo del costo de la atención asociado con la resistencia a meticilina fue de $31 \%$ y, el ajustado, de $70 \%$.

Conclusión. Este estudio constituye un respaldo a los tomadores de decisiones para la búsqueda y la financiación de programas de prevención de infecciones causadas por microorganismos resistentes.

Palabras clave: Staphylococcus aureus resistente a meticilina, bacteriemia, costos y análisis de costo, costos de la atención en salud, cuidados intensivos, infección hospitalaria, control de infecciones, Colombia.

http://dx.doi.org/10.7705/biomedica.v34i3.1692

\section{Economic burden of methicillin-resistant Staphylococcus aureus bacteremia in critical care patients in hospitals in Bogotá}

Introduction: Resistant infections, especially those involving the bloodstream, are associated with a greater use of resources. Their estimates are variable and depend on the methodology used. Staphylococcus aureus is the main pathogen isolated in blood in our hospitals. There is no consolidated data about economic implications of methicillin-resistant $S$. aureus infection.

Objective: To describe the cost of care of methicillin-resistant $S$. aureus bacteremia in a reference population from nine hospitals in Bogotá.

\footnotetext{
Contribución de los autores: y metodológica del proyecto

Juan S. Castillo, Aura L. Leal y Andrés L. González: ejecución del proyecto

Andrés L. González: recolección de datos

Juan S. Castillo, Aura L. Leal, Ricardo Sánchez: análisis de datos

Liliana I. Barrero: participación en todas las etapas del proyecto

Todos los autores participaron en la escritura del manuscrito.
}

Juan S. Castillo, Aura L. Leal, Ricardo Sánchez, Jorge A. Cortés y Carlos A. Álvarez: diseño, financiación, gestión, asesoría temática 
Materials y methods: A multicenter cohort study included 204 patients in a 1:1 ratio according to resistance. Direct medical costs were calculated from hospitalization bills, while the bacteremia period was calculated by applying microcosting based on standard fares.

Results: We found no significant differences between groups in demographic and clinical characteristics, except for resistance risk factors. Fifty-three percent of patients died during hospitalization. Hospital stay and total invoiced value during hospitalization were significantly higher in the group with methicillinresistant S. aureus bacteremia. For this group, higher costs in ICU stay, antibiotics use, intravenous fluids, laboratory tests and respiratory support were recorded. A crude increase of $31 \%$ and an adjusted increase of $70 \%$ in care costs associated with methicillin resistance were registered.

Conclusion: Our study supports decision makers in finding and funding infection prevention programs, especially those infections caused by resistant organisms.

Key words: Methicillin-resistant Staphylococcus aureus, bacteremia, costs and cost analysis, health care costs, intensive care, cross infection, infection control, Colombia.

http://dx.doi.org/10.7705/biomedica.v34i3.1692

La estimación de los costos de las infecciones causadas por microorganismos resistentes a los medicamentos, permite un mejor conocimiento de sus implicaciones económicas en las instituciones de salud y orienta a los tomadores de decisiones en la ejecución de medidas para su prevención y control (1).

La creciente presencia de microorganismos resistentes causantes de infección en los servicios de cuidados intensivos, constituye uno de los principales retos de los profesionales de la salud involucrados en la contención de esta problemática en el ámbito hospitalario (2).

Los reportes de algunos sistemas de vigilancia internacional demuestran que $16,3 \%$ de las infecciones que se presentan en las unidades de cuidados intensivos son ocasionadas por Staphyloccocus aureus $(3,4)$. Este microorganismo se asocia con los peores resultados clínicos en distintos grupos de pacientes (5,6). La información de los programas locales de vigilancia señala que $12,2 \%$ de las infecciones en las unidades de cuidados intensivos, especialmente las asociadas con el uso de catéteres venosos centrales, se originan en aislamientos de $S$. aureus (7), lo que lo convierte en uno de los principales problemas de resistencia bacteriana en las instituciones de salud del país (8). La información proveniente de algunos estudios multicéntricos locales sugiere que la resistencia por sí sola no produce los resultados desfavorables, sino también la respuesta

\section{Correspondencia:}

Liliana Isabel Barrero, Grupo para el Control de la Resistencia Bacteriana de Bogotá (GREBO), Calle $31 \mathrm{~N}^{\circ} 33-38$, piso 2 ,

Bogotá, D.C., Colombia

Teléfono/Fax: 2441508

lisamedun@gmail.com

Recibido: 07/06/13; aceptado: 21/03/14 que los servicios de salud dan al proceso infeccioso (9), la cual suele traducirse en una amplia variedad de esfuerzos terapéuticos y de manejo de las secuelas.

Un número creciente de estudios plantea que las infecciones del torrente sanguíneo son uno de los tipos de infección cuya atención demanda más recursos económicos (10-13), lo que subraya la necesidad de conocer las implicaciones económicas de este fenómeno en nuestro medio, especialmente las relacionadas con $S$. aureus resistente a la meticilina (SARM).

Existen varios métodos para determinar el efecto económico de la resistencia, lo cual origina estimaciones heterogéneas, adaptadas exclusivamente al contexto del que provienen (12,14-17). Hay reportes de incrementos entre 1,3 y 2 veces en los costos de atención cuando se comparan pacientes infectados por $S$. aureus resistente a la meticilina con pacientes con $S$. aureus sensible a dicho medicamento (18). En la literatura científica se reporta una duplicación del costo promedio del tratamiento para pacientes en hemodiálisis con bacteriemia por el germen resistente (€ 10.573 Vs. $€ 24.931)$ (19) y otros estudios han llegado a estimar un costo promedio de hasta $€ 11.044$ por episodio (20).

No existe información detallada en el sistema de salud colombiano sobre las consecuencias económicas de la resistencia bacteriana. Se cree que los costos de la infección por SARM son elevados con base en los estudios internacionales $(16,19,20)$, pero no se cuenta con información local sobre su impacto en los recursos del sector de la salud. En el presente estudio se hizo una aproximación a la relación entre la resistencia de $S$. aureus a la meticilina y el costo de la atención 
en un grupo de pacientes atendido en unidades de cuidados intensivos de Bogotá, en el marco de un estudio multicéntrico de cohortes.

\section{Materiales y métodos \\ Diseño, población y muestra}

Se hizo un análisis de costos anidado en un estudio de cohortes en pacientes con bacteriemia por $S$. aureus, atendidos en las unidades de cuidados intensivos de nueve hospitales públicos y privados de alta complejidad de Bogotá con una capacidad instalada de 90 a 630 camas (9).

Los pacientes se emparejaron en la misma institución y año en una relación de 1:1 según la resistencia del germen. Se seleccionó a cada sujeto en el grupo expuesto al microorganismo sensible, empleando una lista de números aleatorios.

El muestreo para el análisis de costos fue a conveniencia e incluyó a todos los pacientes de aquellos hospitales cuya información sobre costos estaba disponible.

\section{Población del estudio}

Los pacientes con bacteriemia por $S$. aureus se seleccionaron del registro de un sistema de vigilancia de resistencia bacteriana de la ciudad entre enero del 2005 y diciembre del 2008. Se incluyeron pacientes mayores de 15 años que hubieran requerido atención en las unidades de cuidados intensivos (quirúrgicas, médicas, cardiovasculares o de quemados). Se aplicaron las definiciones de los Centers for Disease Control and Prevention (CDC) de los Estados Unidos para la clasificación de aquellas infecciones adquiridas en las instituciones (21). Se incluyeron bacteriemias causadas por un único germen y sólo la primera reportada para cada paciente.

\section{Recolección de la información}

Se diseñó un formato de recolección de la información que incluyó los datos demográficos y clínicos del paciente, la estancia hospitalaria y en la unidad de cuidados intensivos, la duración de la bacteriemia y otras variables relacionadas con los recursos facturados durante el período de la bacteriemia, tales como el número de consultas con otros profesionales, los procedimientos quirúrgicos, las imágenes diagnósticas, los exámenes de laboratorio y los medicamentos, entre ellos los antibióticos, y otros elementos requeridos en el proceso de atención.
Se consideró como inicio de la bacteriemia la fecha de solicitud del hemocultivo mediante el cual se detectó el microorganismo y la finalización se determinó de acuerdo con la evolución clínica y microbiológica de cada paciente ratificada por especialistas en enfermedades infecciosas. Cuando no se pudo determinar el fin de la bacteriemia, se incluyeron las unidades de consumo de recursos hasta un máximo de 21 días después de la fecha de inicio de la bacteriemia.

Antes de acopiar la información relacionada con el consumo de recursos durante la bacteriemia, se recolectaron los datos clínicos y epidemiológicos de cada paciente (9), los cuales incluían las enfermedades concomitantes, medidas con el índice de Charlson (22), y su gravedad, estimada mediante la escala de McCabe (23), así como los antecedentes de exposición a procedimientos quirúrgicos, uso de dispositivos, consumo de antibióticos, estancia hospitalaria previa al inicio de la bacteriemia y otros datos pertinentes sobre la hospitalización durante la cual se hubiera presentado el episodio de bacteriemia.

\section{Estimación del costo directo de la atención}

Al no disponer de la información sobre los costos de atención en la mayoría de las instituciones, se utilizaron las facturas como medida sustitutiva de la variable de interés. Se utilizó la perspectiva del tercer pagador y se obtuvo el valor total facturado durante cada hospitalización y el correspondiente al período de la bacteriemia, incluidos los recursos empleados en la atención directa del paciente en el hospital. Debido a la gran variación de las tarifas de las instituciones participantes, se determinaron unidades de consumo para los ítems de interés durante la bacteriemia (días de estancia, medidas terapéuticas, diagnósticas y de soporte), las cuales se tradujeron a los valores estandarizados en los manuales tarifarios más usados en el momento del estudio $(24,25)$.

Entre las unidades que se registraron por igual para ambos grupos durante los periodos de bacteriemia, se incluyeron los días de hospitalización en la unidad de cuidados intensivos o en otros servicios hospitalarios, el número de consultas con otros profesionales, los días de administración de nutrición entérica y parenteral y de oxígeno, la cantidad de antibióticos (de acuerdo con su presentación), el número de bolsas de líquidos intravenosos, los procedimientos quirúrgicos, los exámenes de laboratorio, las imágenes diagnósticas y los medicamentos para el tratamiento de 
la infección. Una vez establecidas las cantidades de estos insumos, se procedió a asignarles el valor de acuerdo con las tarifas de los manuales, multiplicando la cantidad de cada insumo por el valor correspondiente. Para todos los insumos, excepto los medicamentos, se utilizó el manual del Instituto de Seguros Sociales del 2004 y, para los medicamentos, el manual de la Secretaría Distrital de Salud del 2009 (24,25). Finalmente, se sumaron los valores de los insumos consumidos por cada paciente para obtener una aproximación del costo de la atención durante el periodo de la bacteriemia. Todos los valores se expresaron en pesos colombianos corrientes del 2012 y se ajustaron según el índice de precios al consumidor del sector de la salud (26).

\section{Análisis estadístico}

Se hicieron análisis descriptivos de las variables categóricas y cuantitativas. Se usaron tablas de frecuencias y la prueba de ji al cuadrado o el test exacto de Fisher, así como medidas de tendencia central y dispersión según el resultado del test de Shapiro-Wilk, para contrastar la normalidad, y se compararon las diferencias entre las variables continuas utilizando la prueba t de Student o la prueba de suma de rangos de Wilcoxon según la distribución de la variable. Se hicieron análisis estratificados según la supervivencia de los pacientes, considerando el efecto de la disminución de los costos de atención debido a las muertes (27).

Se ajustó un modelo lineal generalizado con distribución gamma y enlace de logaritmo acorde con lo descrito en la literatura científica para variables económicas que no cumplen con los supuestos de la regresión lineal (28). La variable de respuesta fue el costo directo de la atención. Las variables explicativas se seleccionaron entre las características clínicas y demográficas de los pacientes. Las variables con un nivel de significación menor o igual a 0,10 en el modelo de regresión bivariado, se consideraron en la construcción del modelo multivariado.

Todos los análisis estadísticos se efectuaron con el software Stata, versión 12 (Stata Corp., College Station, TX, USA), las pruebas de hipótesis se hicieron a dos colas y las pruebas e intervalos de confianza se interpretaron con un nivel de significancia de $5 \%$.

\section{Resultados}

Se incluyeron 204 pacientes, 63,2\% de los cuales eran hombres. La mediana de edad fue de 69 años en las mujeres y de 57 años en hombres. No se encontraron diferencias significativas entre los grupos con respecto a la edad, el sexo, la clasificación de McCabe, el puntaje de Charlson y el foco de infección. La proporción de personas con antecedentes de consumo de antibióticos, de cirugías o de uso de dispositivos invasivos antes de la bacteriemia fue significativamente mayor en el grupo de pacientes con bacteriemia causada por SARM (cuadro 1). El $82 \%$ de las bacteriemias se contrajo durante la hospitalización. En el cuadro 2 se resumen los principales resultados clínicos y la comparación de los costos según la resistencia del microorganismo.

\section{Mortalidad}

El $53 \%$ de los sujetos falleció durante la hospitalización. La mortalidad durante la estancia hospitalaria fue mayor en el grupo con el germen resistente $(60,7$ Vs. $45,1 \%)$, al igual que la mortalidad atribuible a la bacteriemia.

\section{Estancia}

Tanto la estancia hospitalaria total como la estancia previa al inicio de la bacteriemia, fueron significativamente mayores en el grupo de pacientes con SARM. La diferencia de siete días en las medianas de estancia previa a la bacteriemia se incrementó a nueve días en las medianas de estancia posterior a esta.

\section{Costos}

El valor total facturado por concepto de la hospitalización fue significativamente mayor en el grupo de pacientes con infección por SARM. Cuando se analizaron solo los pacientes que sobrevivieron, se registraron diferencias en el costo por estancia hospitalaria posterior a la bacteriemia, por estancia en la unidad de cuidados intensivos y en los asociados al tratamiento de la infección. En los pacientes con bacteriemia por $S$. aureus resistente que sobrevivieron, se encontró una diferencia de seis millones de pesos en el valor de la atención del episodio de bacteriemia. Fue evidente que en la atención de estos pacientes se usaron más antibióticos y de mayor costo que en el grupo con el germen sensible (\$2'246.700 Vs. \$431.200), y que se les prescribieron más exámenes de laboratorio clínico, sesiones de terapia respiratoria y líquidos intravenosos (cuadro 2).

En el modelo lineal generalizado bivariado se encontró que la resistencia a la meticilina, la presencia de sepsis grave y la corrección 
Cuadro 1. Características de los pacientes con bacteriemia por Staphylococcus aureus según resistencia a la meticilina

\begin{tabular}{|c|c|c|c|}
\hline Características & $\begin{array}{c}\text { SASM } \\
(n=102)\end{array}$ & $\begin{array}{l}\text { SARM } \\
(n=102)\end{array}$ & p \\
\hline Edad en años cumplidos al ingreso*, mediana (RIC) & $58(32)$ & $63(32)$ & $0,80 \S$ \\
\hline Sexo (hombres) (número - \%) & $61(59,8)$ & $68(66,6)$ & 0,31 \\
\hline Asegurado (subsidiado/vinculado) (número - \%) & $52(50,9)$ & $60(58,8)$ & 0,26 \\
\hline Remitido de otro hospital (\%) & $50(49,02)$ & $51(50)$ & 0,89 \\
\hline Bacteriemia adquirida en otra institución (\%) & $7(6,8)$ & $8(7,8)$ & 0,79 \\
\hline Paciente con alguna condición inmunosupresora (número - \%) & $45(44,1)$ & $53(51,9)$ & 0,26 \\
\hline Charlson, mediana (RIC) & $4(4)$ & $4(6)$ & $0,73 \S$ \\
\hline McCabe, puntaje & & & 0,28 \\
\hline No mortal & $50(49,0)$ & $59(57,8)$ & \\
\hline Finalmente mortal & $46(45,1)$ & $35(34,3)$ & \\
\hline Progresión rápida a muerte & $6(5,8)$ & $8(7,8)$ & \\
\hline Sitio primario (foco) de la infección (número - \%) & & & 0,29 \\
\hline a) Sin foco específico & $32(31,3)$ & $37(36,2)$ & \\
\hline b) Catéter venoso central & $31(30,3)$ & $39(38,2)$ & \\
\hline c) Vías respiratorias & $18(17,6)$ & $12(11,7)$ & \\
\hline d) Absceso o herida quirúrgica & $8(7,8)$ & $3(2,9)$ & \\
\hline e) Otros & $13(12,7)$ & $11(10,7)$ & \\
\hline Bacteriemia de origen hospitalario (número - \%) & $82(80,3)$ & $84(82,3)$ & 0,71 \\
\hline Bacteriemia asociada a la UCI (número - \%) & $54(52,9)$ & $64(62,7)$ & 0,15 \\
\hline Exposición a antibiótico previa a la bacteriemia (hospitalaria y externa) & $29(40,2)$ & $78(83,8)$ & $<0,001$ \\
\hline Cambio de antibiótico (número - \%) & $56(57,1)$ & $38(40)$ & 0,017 \\
\hline Tratamiento inicial apropiado (número - \%) & $82(83,7)$ & $49(51)$ & $<0,001$ \\
\hline Antecedente de cirugía (número - \%) & $51(50)$ & $67(65,7)$ & 0,023 \\
\hline Uso de dispositivos invasivos previo a la bacteriemia (número - \%) & $85(83,3)$ & $98(96,0)$ & 0,003 \\
\hline Hospitalización previa en el último año (número - \%) & $50(49,0)$ & $50(49,0)$ & 1 \\
\hline
\end{tabular}

*: mayores de 15 años

SASM: Staphylococcus aureus sensible a la meticilina

SARM: Staphylococcus aureus resistente a la meticilina

RIC: rango intercuartílico

UCl: unidad de cuidados intensivos

$\S$ Contraste con prueba U de Mann-Whitney

del esquema de antibióticos fueron factores asociados con el incremento en el costo directo de la atención (cuadro 3). Por otro lado, la presencia de enfermedades concomitantes fue un factor que redujo el costo y se asoció con la mortalidad (mediana de enfermedades concomitantes, 2, Vs. 5 en quienes sobrevivieron y quienes murieron). Se observó un incremento significativo de 1,31 en el costo de la atención asociada con la resistencia a la meticilina $\left(\mathrm{IC}_{95 \%} 1,05-1,64\right)$, el cual aumentó a $1,7 \quad\left(\mathrm{IC}_{95 \%}, 1,36-2,12\right)$ una vez se ajustó por otras variables de interés. La presencia de sepsis grave, el uso del tratamiento inicial apropiado o el cambio del esquema inicial, se relacionaron con el incremento del costo.

\section{Discusión}

En el presente estudio se encontró un incremento significativo en el costo de la atención relacionado con la presencia de la resistencia a la meticilina en los pacientes con bacteriemias causadas por $S$. aureus. Los factores que prolongaron la supervivencia, como el uso del tratamiento adecuado o el cambio del esquema de antibióticos durante el seguimiento, se asociaron con un incremento en el costo de la atención. Otro factor que incrementó el costo fue el choque séptico por la demanda subsiguiente de soporte cardiometabólico.

Se reconoce que las infecciones asociadas a la atención en salud son una amenaza para la seguridad de los pacientes (29) y que, cuando son prevenibles, constituyen un costo importante para los servicios sanitarios. El impacto clínico en la mortalidad y la morbilidad de la infección causada por microorganismos resistentes en pacientes gravemente enfermos (9), va de la mano con el incremento en los costos de la atención $y$, probablemente, está asociado a los factores que permiten una mayor supervivencia; en este contexto, los costos más altos se generan por la atención en la unidad de cuidados intensivos y por el consumo de medicamentos e insumos, entre ellos los antibióticos (razón de medianas: 
Cuadro 2. Análisis bivariado de la resistencia a la meticilina y los resultados clínicos y económicos de la bacteriemia

\begin{tabular}{|c|c|c|c|}
\hline Resultados & $\begin{array}{c}\text { SASM } \\
(n=102)\end{array}$ & $\begin{array}{c}\text { SARM } \\
(n=102)\end{array}$ & $\mathbf{p}$ \\
\hline \multicolumn{4}{|l|}{ Clínicos } \\
\hline Estancia hospitalaria total en días, mediana (RIC) & $21 \quad(23)$ & $30(33)$ & $0,0062 \S$ \\
\hline Estancia previa a la bacteriemia (días), mediana (RIC) & $4 \quad(7)$ & 11 (18) & $<0,001 \S$ \\
\hline Estancia posterior a la bacteriemia (días), mediana (RIC)* & $17,5(23)$ & $26,5(27)$ & $0,055 \S$ \\
\hline Mortalidad hospitalaria (estado al egreso) (número - \%) & $46 \quad(45,1)$ & $62(60,7)$ & 0,024 \\
\hline $\begin{array}{l}\text { Mortalidad atribuible a la bacteriemia durante la hospitalización } \\
\text { (directa o probable) (número - \%) }\end{array}$ & $27 \quad(26,4)$ & $45(44,1)$ & 0,008 \\
\hline \multicolumn{4}{|l|}{ Económicos } \\
\hline Total facturado por la hospitalización & $\begin{array}{c}24 ’ 412.699 \\
\left(31^{\prime} 830.380\right)\end{array}$ & $\begin{array}{l}52 ' 243.391 \\
(61 ' 193.350)\end{array}$ & $0,0004 \S$ \\
\hline Total facturado por la hospitalización* & $\begin{array}{l}22{ }^{\prime} 094.247 \\
(32 \cdot 779.472)\end{array}$ & $\begin{array}{c}52,769.281 \\
\left(54^{\prime} 506.484\right)\end{array}$ & $0,0004 \S$ \\
\hline Valor del período de la bacteriemia & $\begin{array}{r}9^{\prime} 731.570 \\
\left(11^{\prime} 663.818\right)\end{array}$ & $\begin{array}{l}11^{\prime} 458.068 \\
\left(17^{\prime} 586.490\right)\end{array}$ & $0,1414 \S$ \\
\hline Valor del período de la bacteriemia* & $\begin{array}{r}9 ’ 397.832 \\
(10 ’ 826.889)\end{array}$ & $\begin{array}{c}16^{\prime} 318.106 \\
\left(17^{\prime} 882.641\right)\end{array}$ & $0,001 \S$ \\
\hline Valor de la estancia en UCI durante la bacteriemia* & $\begin{array}{l}4 ' 266.800 \\
\left(5^{\prime} 120.160\right)\end{array}$ & $\begin{array}{l}6^{\prime} 400.200 \\
\left(8^{\prime} 106.920\right)\end{array}$ & $0,013 \S$ \\
\hline $\begin{array}{l}\text { Valor del consumo de medicamentos durante la bacteriemia } \\
\text { (antibióticos, inotrópicos, líquidos, otros) }{ }^{*}\end{array}$ & $\begin{array}{l}1 ' 170.488 \\
(2,669.715)\end{array}$ & $\begin{array}{l}3,482.798 \\
\left(4^{\prime} 961.731\right)\end{array}$ & $0,0002 \S$ \\
\hline Valor de los antibióticos durante la bacteriemia* & $\begin{array}{r}486.429 \\
\left(1^{\prime} 602.724\right)\end{array}$ & $\begin{array}{l}2 \cdot 746.825 \\
\left(4^{\prime} 034.305\right)\end{array}$ & $0,0001 \S$ \\
\hline Valor de los líquidos durante la bacteriemia* & $\begin{array}{c}58.773 \\
(99.665)\end{array}$ & $\begin{array}{r}92.672 \\
(118.843)\end{array}$ & $0,014 \S$ \\
\hline Valor de exámenes de laboratorio durante la bacteriemia* & $\begin{array}{l}752.263 \\
(693.382)\end{array}$ & $\begin{array}{c}1 ' 304.704 \\
(1 ' 782.487)\end{array}$ & $0,009 \S$ \\
\hline Valor de imágenes diagnósticas durante la bacteriemia* & $\begin{array}{l}208.563 \\
(311.511)\end{array}$ & $\begin{array}{c}223.894 \\
(551.255)\end{array}$ & $0,743 \S$ \\
\hline Valor de los servicios profesionales (consultas) durante la bacteriemia* & $0 \quad(59.250)$ & $0(88.875)$ & $0,851 \S$ \\
\hline Valor de los procedimientos médicos y quirúrgicos durante la bacteriemia* & $0(258.260)$ & $0(657.684)$ & $0,238 \S$ \\
\hline Valor del oxígeno durante la bacteriemia* & $\begin{array}{c}514.368 \\
(952.128)\end{array}$ & $\begin{array}{c}1 ' 026.000 \\
(1 ' 212.048)\end{array}$ & $0,369 \S$ \\
\hline Valor de la terapia respiratoria durante la bacteriemia* & $\begin{array}{c}145.160 \\
(377.416)\end{array}$ & $\begin{array}{c}304.836 \\
(544.350)\end{array}$ & $0,028 \S$ \\
\hline
\end{tabular}

* Análisis en población superviviente (SASM=56 y SARM=40)

RIC: rango intercuartílico

UCI: unidad de cuidados intensivos

$\S$ Contraste con prueba U de Mann-Whitney

Todos los valores en pesos del 2012

5,65 veces al comparar el costo de los antibióticos para pacientes con SARM y el de pacientes con $S$. aureus sensible a la meticilina).

En un estudio español se comprobó en los pacientes con SARM una mayor utilización de antibióticos, una estancia más prolongada, más utilización de pruebas diagnósticas y mayores tasas de ingreso a la unidad de cuidados intensivos (20). En otro estudio en los Estados Unidos se encontraron diferencias en el costo medio, el costo de la atención hospitalaria posterior a la bacteriemia y durante la estancia en el hospital, siendo mayores en el grupo de pacientes con bacteriemia por SARM, diferencias que, sin embargo, se desvanecieron al ajustar los valores por medio del análisis multivariado $o$ análisis de propensión (30). En este último estudio se refleja la importancia de ajustar por las diferentes variables que modifican el costo (por ejemplo, la admisión en la unidad de cuidados intensivos). Teniendo en cuenta las diferencias entre los sistemas de salud, es importante, además, verificar el impacto de la resistencia a nivel local.

Se cree que los pacientes con infecciones causadas por microorganismos resistentes presentan en ocasiones un mayor número de enfermedades concomitantes o cuadros clínicos más graves, una mortalidad más temprana y estancias hospitalarias 
Cuadro 3. Impacto económico en el valor facturado por la atención asociada con la resistencia a meticilina y otras características clínicas de los pacientes con bacteriemia

\begin{tabular}{|c|c|c|c|c|c|c|}
\hline \multirow{3}{*}{$\begin{array}{l}\text { Factor relacionado } \\
\text { Asegurado* }\end{array}$} & \multicolumn{3}{|c|}{ Análisis bivariado } & \multicolumn{3}{|c|}{ Análisis multivariado } \\
\hline & \multirow{2}{*}{$\begin{array}{c}\begin{array}{c}\text { Razón ajustada } \\
\text { de costos }\end{array} \\
1,06\end{array}$} & \multicolumn{2}{|c|}{$\begin{array}{c}\text { Intervalo de } \\
\text { confianza } 95 \%\end{array}$} & \multirow[t]{2}{*}{$\begin{array}{l}\text { Razón ajustada } \\
\text { de costos }\end{array}$} & \multicolumn{2}{|c|}{$\begin{array}{c}\text { Intervalo de } \\
\text { confianza } 95 \%\end{array}$} \\
\hline & & 0,84 & 1,35 & & & \\
\hline Cambio de antibiótico & 1,62 & 1,3 & 2,02 & 1,58 & 1,23 & 2,02 \\
\hline Choque séptico & 1,13 & 0,9 & 1,43 & & & \\
\hline Enfermedades concomitantes (Charlson) & 0,96 & 0,92 & 0,99 & 0,96 & 0,92 & 0,99 \\
\hline Resultado (muerte) & 0,88 & 0,7 & 1,12 & & & \\
\hline Edad (año) & 1 & 0,99 & 1,01 & & & \\
\hline Fuente de infección eliminable & 1,21 & 0,96 & 1,54 & & & \\
\hline Gravedad al inicio (APACHE II) & 0,99 & 0,98 & 1,01 & & & \\
\hline Gravedad al inicio (McCabe) & 1,07 & 0,9 & 1,28 & & & \\
\hline Resistencia a la meticilina & 1,31 & 1,05 & 1,64 & 1,7 & 1,36 & 2,12 \\
\hline Sepsis grave & 1,35 & 1,03 & 1,76 & 1,57 & 1,23 & 2,01 \\
\hline Sexo (hombre) & 1,14 & 0,89 & 1,46 & & & \\
\hline Tratamiento inicial inapropiado & 1,09 & 0,85 & 1,4 & 2,08 & 1,67 & 2,59 \\
\hline
\end{tabular}

* Régimen contributivo, prepagado y de pago privado Vs. subsidiado y vinculado

previas más prolongadas. Esta consideración exige que los análisis para determinar la relación entre la resistencia y los resultados, tales como la prolongación de la estancia hospitalaria y el aumento de los costos, incluyan ajustes por gravedad y mortalidad prematura, lo cual limita el seguimiento, para no sobrestimar los valores. En la población del presente estudio, la presencia de enfermedades concomitantes fue el único factor asociado con la reducción del costo en razón de la mortalidad prematura.

No se registraron diferencias significativas en las características demográficas o clínicas de base de la población observada; se encontró que el grupo de pacientes con bacteriemia causada por el germen resistente había tenido estancias hospitalarias previas más prolongadas, así como mayor exposición a antibióticos y procedimientos invasivos, todos ellos factores de riesgo descritos en la bibliografía científica internacional y nacional $(6,9)$.

El incremento registrado en el costo de la atención derivado de una mayor supervivencia no constituye un problema para el sistema de salud, en tanto que la atención de la infección por microorganismos resistentes, el manejo inicial inadecuado o la detección tardía de los pacientes resistentes, sí representan costos innecesarios. Estos resultados plantean dos reflexiones. La primera se relaciona con la necesidad de una atención temprana de los pacientes para evitar cualquier tipo de infección, ya sea por microorganismos resistentes o sensibles; la segunda se refiere a la necesidad de mantener un equilibrio entre el diagnóstico y el tratamiento apropiados, que garantice los mejores resultados clínicos.

El carácter privado del 'costeo' y de la definición de las tarifas en las diferentes instituciones impide comparar fácilmente los resultados entre estas y entre los diferentes escenarios de atención en salud. La técnica de 'costeo' basada en actividades, empleada para aproximar el costo de la atención durante el período de la bacteriemia, permite comparar diferencias entre los grupos de acuerdo con la exposición a un germen sensible o resistente, sin tener en cuenta el tiempo previo a dicha exposición o la institución de procedencia de los pacientes. Los costos estandarizados de acuerdo con una misma tarifa representan una fortaleza del trabajo que permite agregar información proveniente de instituciones con diferentes sistemas de costos. Aunque en el estudio no se calculó el tamaño de la muestra, se estimó que esta tenía un poder de más de 90 \% para encontrar la diferencia de costos observada.

Existen diferentes estrategias de estimación de los costos de la atención de los pacientes con infecciones (12,14-17). Sin embargo, nuestro sistema de salud aún no cuenta con bases de datos y sistemas contables comparables que faciliten el uso de las técnicas reconocidas como las más apropiadas, por ejemplo, la herramienta razón costo-factura (charge to cost ratio) empleada en Norteamérica (31). 
Entre las limitaciones más importantes para la validez del estudio se encuentran la calidad de la información disponible en los sistemas de registro y facturación de las instituciones participantes y la posibilidad de un sesgo de selección al haber incluido pacientes con diagnóstico microbiológico confirmado o infecciones por SARM que solo se pudieron emparejar con pacientes afectados por bacteriemias por $S$. aureus sensible de la misma institución. Otra limitación importante fue la ausencia de un grupo de comparación sin infección que diera cuenta del impacto económico real de la infección por S. aureus.

No obstante estas limitaciones, se logró una aproximación al costo de la presencia de SARM en bacteriemias para nuestro sistema de salud. Los resultados muestran un incremento crudo de $31 \%$ y uno ajustado de $70 \%$ en el costo de la atención asociada con la resistencia a la meticilina. Nuestros resultados coinciden con los de otros estudios publicados que emplean aproximaciones más conservadoras al problema (16). Este estudio constituye un respaldo para los tomadores de decisiones en la búsqueda de programas orientados a la prevención de la infección, en especial, la causada por microorganismos resistentes.

\section{Agradecimientos}

A todo el personal de apoyo y al personal de los servicios de estadística y archivo de las instituciones que participaron en el estudio.

\section{Conflicto de intereses}

Ninguno de los autores declara tener conflictos de intereses en la realización del presente estudio.

\section{Financiación}

Este estudio se llevó a cabo con subvenciones para la investigación del Departamento Administrativo de Ciencia, Tecnología e Innovación, Colciencias, (110140820452-2007) y de la Universidad Nacional de Colombia (DIB-2008 202010011672). Además, contó con el respaldo científico de la Asociación Colombiana de Infectología, Capítulo Central $(A C I N)$, y de la Secretaría Distrital de Salud de Bogotá, Área de Vigilancia en Salud Pública.

Instituciones que conforman el grupo GREBO

Hospital Simón Bolívar, E.S.E. (Felipe Zamora), Fundación Hospital San Carlos (Jaime Saravia, Yaníz Hernández), Hospital El Tunal, E.S.E. (Narda Olarte, Alberto Valderrama), Hospital de Occidente Kennedy (Andrés Almánzar, Carlos
Alquichire), Fundación Cardio-Infantil (Álvaro Arango, Zenaida Montañez), Hospital Universitario Clínica San Rafael (Carlos Saavedra, Henry Oliveros), Hospital Universitario de La Samaritana (Carlos Pérez, Beatriz Cuevas, Nancy Osorio), Hospital Santa Clara, E.S.E. (Ibeth Pernet, Claudia Cuéllar), Hospital Universitario Mayor (Sonia Castañeda), Universidad Nacional de Colombia (Sonia Romero), Secretaría Distrital de Salud (María Patricia González), Asociación Colombiana de Infectología - Capítulo Central.

\section{Referencias}

1. Cohen B, Larson EL, Stone PW, Neidell M, Glied SA. Factors associated with variation in estimates of the cost of resistant infections. Med Care. 2010;48:767-75. http:// dx.doi.org/10.1097/MLR.0b013e3181e358b9

2. Cosgrove $\mathbf{S}$. The relationship between antimicrobial resistance and patient outcomes: Mortality, length of hospital stay, and health care costs. Clin Infect Dis. 2006;42:S82-9. http://dx.doi.org/10.1086/499406

3. Wisplinghoff $H$, Bischoff T, Tallent SM, Seifert $H$, Wenzel RP, Edmond MB. Nosocomial bloodstream infections in US hospitals: Analysis of 24,179 cases from a prospective nationwide surveillance study. Clin Infect Dis. 2004;39:30917. http://dx.doi.org/10.1086/421946

4. Gastmeier P, Geffers C, Sohr D, Dettenkofer M, Daschner F, Ruden H. Five years working with the German nosocomial infection surveillance system (Krankenhaus Infektions Surveillance System). Am J Infect Control. 2003;31:316-21. http://dx.doi.org/10.1067/mic.2003.66

5. Whitby M, McLaws ML, Berry G. Risk of death from methicillin-resistant Staphylococcus aureus bacteraemia: A meta-analysis. Med J Aust. 2001;175:264-7.

6. Cosgrove SE, Sakoulas G, Perencevich EN, Schwaber MJ, Karchmer AW, Carmeli Y. Comparison of mortality associated with methicillin-resistant and methicillinsusceptible Staphylococcus aureus bacteremia: A metaanalysis. Clin Infect Dis. 2003;36:53-9. http://dx.doi.org/10. $1086 / 345476$

7. Granados M, Londoño H, Vargas M, Arango J, Benítez F, Barciela E, et al. Epidemiología de la bacteriemia asociada a catéteres endovasculares en 35 unidades de cuidados intensivos de Colombia (2007-2008). Acta Colombiana de Cuidado Intensivo. 2009;9:36-42.

8. Secretaría Distrital de Salud de Bogotá. Boletín Epidemiológico Distrital de Infecciones asociadas al cuidado de la salud, 2010. Fecha de consulta: 21 de diciembre de 2012. Disponible en: http://www.saludcapital.gov.co/sitios/ VigilanciaSaludPublica/Todo\%20IIH/Bolet\%C3\%ADn\%20 SIVIBAC\%202010.pdf.

9. Castillo JS, Leal AL, Cortés JA, Álvarez CA, Sánchez R, Buitrago G, et al. Mortality among critically ill patients with methicillin-resistant Staphylococcus aureus bacteremia: A multicenter cohort study in Colombia. Rev Panam Salud Pública. 2012;32:343-50. http://dx.doi.org/10.1590/S102049892012001100004

10. Pittet D, Tarara D, Wenzel RP. Nosocomial bloodstream infection in critically ill patients. Excess length of stay, extra 
costs, and attributable mortality. JAMA. 1994;271:1598-601. http://dx.doi.org/10.1001/jama.1994.03510440058033

11. Digiovine B, Chenoweth C, Watts C, Higgins M. The attributable mortality and costs of primary nosocomial bloodstream infections in the intensive care unit. Am J Respir Crit Care Med. 1999:160:976-81. http://dx.doi.org/10.1164/ ajrccm.160.3.9808145

12. Stone PW, Braccia D, Larson E. Systematic review of economic analyses of health care-associated infections. Am J Infect Control. 2005;33:501-9. http://dx.doi.org/10.1016/j. ajic.2005.04.246

13. Scott RD 2nd. The direct medical costs of health care associated infections in US hospitals and the benefits of prevention. Centers for Disease Control and Prevention. 2009. Fecha de consulta: 2 de julio de 2010. Disponible en: http://www.cdc.gov/hai/pdfs/hai/scott_costpaper.pdf.

14. Graves N, Harbarth S, Beyersmann J, Barnett A, Halton K, Cooper B. Estimating the cost of health careassociated infections: Mind your p's and q's. Clin Infect Dis. 2010;50:1017-21. http://dx.doi.org/10.1086/651110

15. Barnett AG, Batra R, Graves N, Edgeworth J, Robotham J, Cooper B. Using a longitudinal model to estimate the effect of methicillin-resistant Staphylococcus aureus infection on length of stay in an intensive care unit. Am J Epidemiol. 2009;170:1186-94. http://dx.doi.org/10.1093/aje/kwp249

16. Resch A, Wilke M, Fink C. The cost of resistance: Incremental cost of methicillin-resistant Staphylococcus aureus (MRSA) in German hospitals. Eur J Health Econ. 2009;10:287-97. http://dx.doi.org/10.1007/s10198-008-0132-3

17. Riu M, Terradas $\mathbf{R}$, Sala $\mathbf{M}$, Comas $\mathbf{M}$, Knobel $\mathbf{H}$, Grau S, et al. Costs associated with nosocomial bacteraemias in a University Hospital. Enferm Infecc Microbiol Clin. 2012;30:137-42. http://dx.doi.org/10.1016/j. eimc.2011.11.006

18. Cosgrove SE, Carmeli Y. The impact of antimicrobial resistance on health and economic outcomes. Clin Infect Dis. 2003;36:1433-7. http://dx.doi.org/10.1086/375081

19. Greiner W, Rasch A, Köhler D, Salzberger B, Fätkenheuer G, Leidig M. Clinical outcome and costs of nosocomial and community-acquired Staphylococcus aureus bloodstream infection in haemodialysis patients. Clin Microbiol Infect. 2007;13:264-8.http://dx.doi.org/10.1111/j.1469-0691.2006. 01622.x

20. Rubio-Terres C, Garau J, Grau S, Martínez-Martínez L. Cost of bacteraemia caused by methicillin-resistant $V s$. methicillin-susceptible Staphylococcus aureus in Spain: A retrospective cohort study. Clin Microbiol Infect. 2010;16: 722-8. http://dx.doi.org/10.1111/j.1469-0691.2009.02902.x

21. Horan TC, Andrus M, Dudeck MA. CDC/NHSN surveillance definition of health care.associated infection and criteria for specific types of infections in the acute care setting. Am J Infect Control. 2008;36:309-32. http://dx.doi.org/10.1016/j. ajic.2008.03.002

22. Charlson ME, Pompei P, Ales KL, MacKenzie CR. A new method of classifying prognostic comorbidity in longitudinal studies: Development and validation. J Chronic Dis. 1987;40:373-83. http://dx.doi.org/10.1016/0021-9681(87) 90171-8

23. Craven DE, Kunches LM, Lichtenberg DA, Kollisch NR, Barry MA, Heeren TC, et al. Nosocomial infection and fatality in medical and surgical intensive care unit patients. Arch Intern Med. 1988;148:1161-8. http://dx.doi. org/10.1001/archinte.1988.00380050165024

24. Imprenta Nacional de Colombia. Diario Oficial No. 45478 del 2 de marzo de 2004. Manual de tarifas de la entidad promotora de salud del seguro social "EPS-ISS". Acuerdo No. 312 de 2004. Fecha de consulta: 22 de febrero de 2014. Disponible en: http://www.imprenta.gov.co/portal/page/ portal/IMPRENTA/Productos/Diario_Oficial.

25. Secretaría Distrital de Salud. Manual Tarifario de Medicamentos, 2009. Fecha de consulta: 22 de febrero de 2014. Disponible en: http://www.saludcapital.gov.co/DDS/ Paginas/ManualTarifario.aspx.

26. Departamento Administrativo Nacional de Estadística. Índice de precios al consumidor. Certificación de IPC con firma digital. Fecha de consulta: 22 de febrero de 2014. Disponible en: http://www.dane.gov.co/index.php/indicesde-precios-y-costos/indice-de-precios-al-consumidor-ipc.

27. Organización Panamericana de la Salud. Protocolo para determinar el costo de la infección intrahospitalaria. Fecha de consulta: 2 de mayo de 2013. Disponible en: www.paho. org/Spanish/AD/DPC/CD/protocolo.pdf.

28. Manning WG, Basu A, Mullahy J. Generalized modeling approaches to risk adjustment of skewed outcomes data. J Health Econ. 2005;24:465-88. http://dx.doi.org/10.1016/j. jhealeco.2004.09.011

29. World Health Organization. Guidelines on hand hygiene in health care, 2009. Fecha de consulta: 2 de mayo de 2013. Disponible en: http://whqlibdoc.who.int/ publications/2009/9789241597906_eng.pdf?ua=1.

30. Ben-David D, Novikov I, Mermel LA. Are there differences in hospital cost between patients with nosocomial methicillinresistant Staphylococcus aureus bloodstream infection and those with methicillin-susceptible S. aureus bloodstream infection? Infect Control Hosp Epidemiol. 2009;30:453-60. http://dx.doi.org/10.1086/596731

31. Drummond M, O'Brien B, Stoddart G, Torrance G. Métodos para la evaluación económica de los programas de asistencia sanitaria. $2^{\underline{a}}$ edición. Madrid: Ediciones Díaz de Santos; 2001. 